\title{
High Energy Cocktail Beams for Radiation Effects Studies*
}

\author{
M.A. McMahan, D. Argento, T. Gimpel, A. Guy, J. Morel, K. Osborne, R. Siero, R. Thatcher, D. Wutte, X.Q.
} Xie* ${ }^{*}$ C.M. Lyneis

The 88-Inch Cyclotron facility consists of a sector-focussed, variable energy cyclotron fed by two Electron Cyclotron Resonance (ECR) high charge-state sources. The combination of cyclotron and ECR source provides the ability to run "cocktails" of ions, mixtures of ions of nearidentical charge-to-mass ratio. This concept was developed for detector calibrations soon after the first ECR source came on line. [1] The ions are tuned out of the source together and the cyclotron frequency -acting as a mass analyzer - separates them. This means that within the components of the cocktail, the accelerated ion can be changed in approximately one minute. Intensity variations are compensated for with a series of attenuator grids at the ion source, which allow ion beam intensity adjustments over nine orders of magnitude.

The cocktail beam soon became the mainstay of the heavy ion radiation effects program at the Cyclotron, an applied program which is run on a recharge basis and which accounts for about $10 \%$ of the annual usage. The largest component of this program is directed at measuring single event effects - a variety of effects, which can occur when a heavy ion traverses a transistor, memory chip or other piece of microelectronics. It can then be determined whether the device is appropriate for use in a satellite or space vehicle in a particular orbit, knowing the distribution of heavy ions expected at that orbit.

To first order, the observed single event effects are atomic processes, which depend only on the linear energy transfer (LET), or stopping power, of the ion. The range of the ion is important only to ensure that the ion reaches the correct interaction depth. In the early 90 's, two cocktails were developed at the Cyclotron for standard use: a "light ion" cocktail for ions up to ${ }^{36} \mathrm{Ar}$ at high energy and large range, and a "heavy ion" cocktail at $4.5 \mathrm{MeV} /$ nucleon, with LETs ranging from 3-60
$\mathrm{MeV} / \mathrm{mg} / \mathrm{cm}^{2}$ and ranges from $43-69 \mu$. Using the AECR, Bi could be added, giving a LET of nearly $100 \mathrm{MeV} / \mathrm{mg} / \mathrm{cm}^{2}$.

In recent years, as chip technology advanced and chips became more miniaturized, it has become more difficult to "delid" the chips, i.e. remove the layers on top of the active elements to allow the ions to penetrate to the active area. Therefore many users were requesting a higher energy heavy-ion cocktail. With the upgrade of the Advanced ECR source in 1996 and vacuum upgrades to the Cyclotron in subsequent years, it became possible to accelerate a cocktail of beams at $10 \mathrm{MeV} / \mathrm{u}$. This gives LETs not very different than the lower energy cocktail, 0.8-53 $\mathrm{MeV} / \mathrm{mg} / \mathrm{cm}^{2}$, and ranges from $115-330 \mu$. The "standard" components of this cocktail are given in Table 1. Other beams ( $\mathrm{Mg}, \mathrm{Ge}, \mathrm{Mo})$ can be found at lower intensities. This cocktail now accounts for approximately $50 \%$ of the radiation effect work done at the 88" Cyclotron.

Table 1. $10 \mathrm{MeV} / \mathrm{u}$ cocktail

\begin{tabular}{|l|c|c|c|}
\hline Ion & $\begin{array}{c}\text { Energy } \\
(\mathrm{MeV})\end{array}$ & $\begin{array}{c}\text { LET } \\
(\mathrm{MeV} / \mathrm{mg} / \mathrm{cm} 2)\end{array}$ & Range $(\mu)$. \\
\hline${ }^{11} \mathrm{~B}^{+3}$ & 108.2 & 0.89 & 323 \\
\hline${ }^{18} \mathrm{O}^{+5}$ & 183.5 & 2.19 & 228 \\
\hline${ }^{22} \mathrm{Ne}^{+6}$ & 216.3 & 3.44 & 179 \\
\hline${ }^{40} \mathrm{Ar}^{+11}$ & 400. & 9.88 & 323 \\
\hline${ }^{51} \mathrm{~V}^{+14}$ & 508.3 & 14.8 & 116 \\
\hline${ }^{65} \mathrm{Cu}^{+18}$ & 659.2 & 21.6 & 108. \\
\hline${ }^{86} \mathrm{Kr}^{+24}$ & 886. & 30.0 & 111. \\
\hline${ }^{136} \mathrm{Xe}^{+38}$ & 1403.4 & 53.6 & 110. \\
\hline
\end{tabular}

Footnotes and References

* Present Address: BIE Berkeley Ion Equipment, Inc., 3400 De La Cruz Building V, Santa Clara, CA 95054

1. M.A. McMahan, et al, Using a Cyclotron plus ECR Source for Detector Evaluation and Calibration, Nucl. Instr. \& Meth. A253, 1 (1986). 